\title{
FET-PET radiomics in recurrent glioblastoma: prognostic value for outcome after re-irradiation?
}

\author{
Montserrat Carles ${ }^{1,2,3^{*}+}$, Ilinca Popp ${ }^{2,4 \dagger}{ }^{4}$ Michael Maximilian Starke ${ }^{4}$, Michael Mix ${ }^{2,5}$, Horst Urbach ${ }^{6}$, \\ Tanja Schimek-Jasch ${ }^{4}$, Franziska Eckert ${ }^{7,8}$, Maximilian Niyazi ${ }^{9,10}$, Dimos Baltas ${ }^{1,2}$ and Anca L. Grosu ${ }^{2,4}$
}

\begin{abstract}
Purpose: The value of O-(2-[18F]fluoroethyl)-L-tyrosine (FET)-positron emission tomography (PET)-radiomics in the outcome assessment of patients with recurrent glioblastoma ( $\mathrm{rGBM}$ ) has not been evaluated until now. The aim of this study was to evaluate whether a prognostic model based on FET-PET radiomics features (RF) is feasible and can identify rGBM patients that would most benefit from re-irradiation.

Methods: We prospectively recruited rGBM patients who underwent FET-PET before re-irradiation (GLIAA-Pilot trial, DRKS00000633). Tumor volume was delineated using a semi-automatic method with a threshold of 1.8 times the standardized-uptake-value of the background. 135 FET-RF (histogram parameters, shape and texture features) were extracted. The analysis involved the characterization of tumor and non-tumor tissue with FET-RF and the evaluation of the prognostic value of FET-RF for time-to-progression (TTP), overall survival (OS) and recurrence location (RL).

Results: Thirty-two rGBM patients constituted our cohort. FET-RF discriminated significantly between tumor and non-tumor. The texture feature Small-Zone-Low-Gray-Level-Emphasis (SZLGE) showed the best performance for the prediction of TTP ( $p=0.001$, satisfying Bonferroni-multiple-test significance level). Additionally, two radiomics signatures could predict TTP (TTP-radiomics-signature, $p=0.001$ ) and OS (OS-radiomics-signature, $p=0.038$ ). SZLGE and the TTP-radiomics-signature additionally predicted RL. Specifically, high values for TTP-radiomics-signature and for SZLGE indicated not only earlier progression, but also a RL within the initial FET-PET active volume.
\end{abstract}

Conclusion: Our findings suggest that FET-PET radiomics could contribute to the prognostic assessment and selection of rGBM-patients benefiting from re-irradiation.

Trial registration DRKS00000633. Registered on 8th of December in 2010.

https://www.drks.de/drks_web/navigate.do?navigationld=trial.HTML\&TRIAL_ID=DRKS00000633.

Keywords: Recurrent-glioblastoma, FET-PET, Radiomics, Re-irradiation

*Correspondence: montserrat.carles@uniklinik-freiburg.de

${ }^{\dagger}$ Montserrat Carles and Ilinca Popp have contributed equally ${ }^{1}$ Division of Medical Physics, Department of Radiation Oncology, Medical Center, University of Freiburg, Robert-Koch Str. 3, 79106 Freiburg, Germany

Full list of author information is available at the end of the article

\section{Introduction}

Surgery, radiation therapy (RT) and chemotherapy is the standard treatment in glioblastoma (GBM) [1]. In recurrent glioblastoma (rGBM), re-irradiation (re-RT) is an important therapeutic alternative, which may delay further disease progression and improve survival $[2,3]$. However, the re-RT can be associated with a high risk of brain edema, radiation necrosis, increased dose of original author(s) and the source, provide a link to the Creative Commons licence, and indicate if changes were made. The images or other third party material in this article are included in the article's Creative Commons licence, unless indicated otherwise in a credit line to the material. If material is not included in the article's Creative Commons licence and your intended use is not permitted by statutory regulation or exceeds the permitted use, you will need to obtain permission directly from the copyright holder. To view a copy of this licence, visit http://creativecommons.org/licenses/by/4.0/. The Creative Commons Public Domain Dedication waiver (http://creativeco mmons.org/publicdomain/zero/1.0/) applies to the data made available in this article, unless otherwise stated in a credit line to the data. 
corticosteroids and impaired quality of life. Therefore, the selection of patients who will most probably benefit from this treatment is extremely important. However, apart from general clinical criteria, such as primary histology, methylation, performance status, time between first and second RT and age $[4,5]$, there are currently only few studies showing that imaging biomarkers could predict treatment outcome after re-RT in rGBM [6-8].

Generally, diagnosis, treatment planning and followup of GBM are based on magnetic resonance imaging (MRI): gadolinium contrast enhanced T1-weighted images (Gd-T1MR), T2 images, FLAIR images etc. Contrast enhancement is facilitated by a disruption of the blood-brain-barrier, highly correlating with malignant tumor tissue. Nevertheless, contrast enhancement could also occur after a recent surgery, RT or chemotherapy (pseudoprogression). In this case, amino-acid positron emission tomography (PET) has been proven to be able to non-invasively differentiate treatment-related changes from real tumor progression [8-10]. Consequently, the use of the radiolabeled amino acid O-(2-[18F] fluoroethyl)-L-tyrosine (FET) has rapidly increased in the last decade [9-14].

Research focused on the application of radiomics at different cancer sites has seen a significant boost in the past years. The aim of radiomics is the development of models based on the analysis of quantitative features (first and higher order statistics) derived from different imaging modalities $[15,16]$. For gliomas, most of the radiomics publications have focused on MRI [17, 18]. Although only few recent studies have involved FET-PET image analysis, they showed the added value of FET-PET in the classification of tumor grades [19] and in predicting IDH genotype [20].

To the best of our knowledge, the prognostic role of FET-PET radiomics in patients with rGBM scheduled for re-irradiation has not yet been evaluated. The aim of the current study was to assess the feasibility of developing a model based on FET radiomics features (RF), with the objective of selecting rGBM patients that would most benefit from re-irradiation.

\section{Materials and methods \\ Patients}

Our study involved patients with rGBM (WHO grade IV) who had undergone standard radiochemotherapy after primary diagnosis. Thirty-two patients were prospectively enrolled in the open label mono-center pilot trial (DRKS00000633) of the GLIAA trial (NOA 10/ARO 2013-1) [21]. The clinical information for the patient cohort is summarized in Table 1. For all patients, the time between the first RT and re-RT was at least 6 months and the recurrent tumor was visible on baseline FET-PET
Table 1 Clinical information of the patient cohort. TTP: time interval between the start of the re-irradiation treatment and the first progression

\begin{tabular}{ll}
\hline & Patient cohort ( $\mathbf{n = 3 2})$ \\
\hline Age (years, median, range) & $52(30-77)$ \\
Sex & \\
Male & $17(53.1 \%)$ \\
Female & $15(46.9 \%)$ \\
GBM & \\
Unifocal & $18(56.3 \%)$ \\
Multifocal & $14(43.7 \%)$ \\
IDH-mutation & \\
Mutated & $10(31.2 \%)$ \\
Wild-type & $14(43.8 \%)$ \\
Unknown & $8(25 \%)$ \\
MGMT-status of recurrent tumor & \\
Methylated & $7(21.9 \%)$ \\
Not methylated & $8(25 \%)$ \\
Unknown & $17(53.1 \%)$ \\
Surgery of recurrent tumor before re-irradia- & \\
Yion & \\
Nes & \\
Time to progression (TTP) (days, median, & $91(18-405)$ \\
\hline
\end{tabular}

OS time interval between the start of re-irradiation and death

with a diameter ranging from 1 to $6 \mathrm{~cm}$. High-precision re-irradiation was performed in a stereotactic setup according to clinic standards. Dose specifications, contouring and constraints for organs at risk were identical to the ongoing GLIAA trial [21]. The prescribed dose was $39 \mathrm{~Gy}, 3 \mathrm{~Gy} / \mathrm{d}, 5 \mathrm{x} /$ week and was set to ensure coverage of at least $95 \%$ of the planning target volume. Progression was diagnosed on conventional MRI in interdisciplinary tumor conferences of the Comprehensive Cancer Center Freiburg, by taking the updated RANO-criteria [22] and the patients' clinical condition into consideration. Additional FET-PET and/or histological confirmation were used in unclear progression cases. Time-to-tumor-progression (TTP) and overall survival (OS) were defined as the times between the start of the re-irradiation treatment and the first progression for TTP and death for OS. Recurrence location (RL) distinguished between inside ( $>50 \%$ volume) or outside the initial FET-PET active volume (see $V_{\text {PET }}$ definition in subsection "Segmentation").

\section{FET-PET imaging}

The ${ }^{18} \mathrm{~F}$-labeled amino acid was synthesized via $\left[{ }^{18} \mathrm{~F}\right]$-fluoroalkylation of tyrosine with a specific activity larger than $18.5 \mathrm{GBq} / \mu \mathrm{mol}$. The FET-PET acquisition protocol for our patient cohort was defined as follows: a 
static 15 min scan was performed 20 min post intravenous injection of 200-300 MBq FET. Scans were performed on two different PET/CT systems from Philips (Netherlands): GEMINI TF TOF 64 (TF64) and GEMINI TF 16 Big Bore (BB). BB was employed for 14 of the 32 patients. The scanners fulfilled the requirements indicated in the European Association of Nuclear Medicine (EANM) imaging guidelines and obtained EANM Research Ltd. (EARL) accreditation. The transverse spatial resolution at $1 \mathrm{~cm}$ from the central axis of the scanner was $4.8 \mathrm{~mm}$. PET data was corrected for random coincidences, scatter and attenuation, based on the corresponding CT dataset. The reconstruction methods was a LOR-based ordered-subset iterative time-offlight algorithm using spherical coordinates (BLOB) with three iterations, 33 subsets and a relaxation parameter for smoothing of 0.35 . PET images had a voxel size of $2 \times 2 \times 2 \mathrm{~mm}^{3}$ and were normalized to decay-corrected injected activity per $\mathrm{kg}$ body weight (standardizeduptake-value SUV $[\mathrm{g} / \mathrm{ml}]$ ).

\section{Segmentation}

Delineation on FET-PET took place in three steps (Fig. 1). Firstly, two spheres of constant diameter were

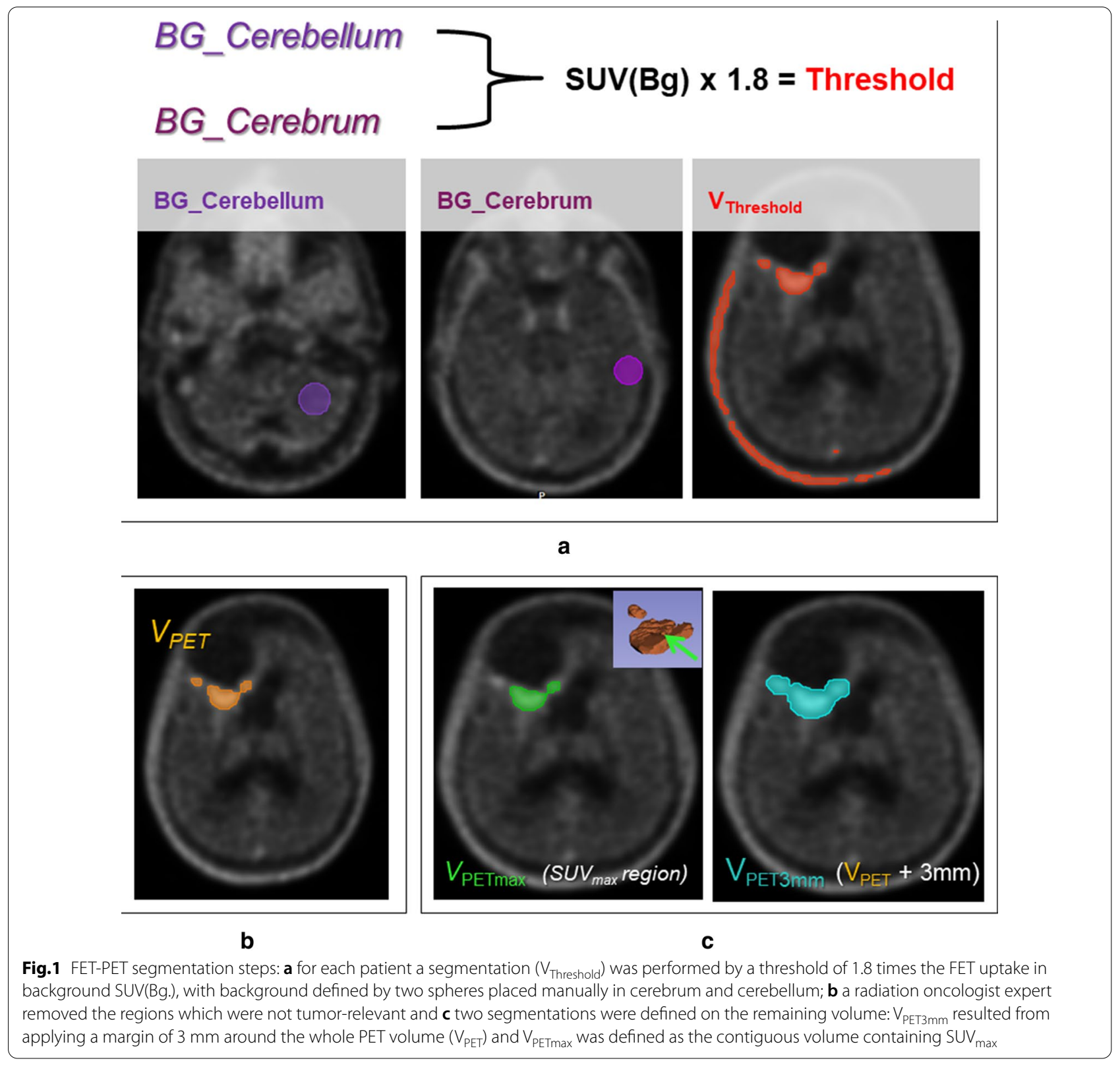


manually placed in cerebrum and cerebellum. SUV of the background, SUV(Bg.), was defined by means of the average SUV values derived from the two spheres. Secondly, a threshold defined as $1.8 \times \mathrm{SUV}(\mathrm{Bg}$.) was applied. From the resulting volume $\left(\mathrm{V}_{\text {Threshold }}\right)$, an experienced radiation oncologist removed the regions that were considered not tumor-related (e.g. blood vessels and extra cerebral enhancement), based on anatomical information conveyed by MRI and CT. Finally, from the remaining volume $\left(\mathrm{V}_{\mathrm{PET}}\right)$ two different contours $\left(\mathrm{V}_{\mathrm{PETmax}}\right.$ and $\left.\mathrm{V}_{\text {PET3mm }}\right)$ were generated. For the volume $V_{\text {PET3mm }}$, a margin of $3 \mathrm{~mm}$ was applied to $\mathrm{V}_{\mathrm{PET}}$. It was defined to cover all regions of FET uptake as well as the surrounding volume that could contain information concerning microscopic tumor cell spread, equivalent to the clinical target volume used for RT. For the high-risk volume defined as $\mathrm{V}_{\text {PETmax }}$, only the contiguous contour containing the maximum SUV was considered. The analysis of the $\mathrm{V}_{\text {PETmax }}$ was based on the hypothesis that the region with higher FETuptake should be the most metabolically and mitotically active one and could therefore provide prognostic information concerning radiotherapy outcome.

\section{Radiomics features extraction}

135 RF were computed using in-house software based on MATLAB $^{\circledR}$ (The MathWorks Inc., Natick, MA). Definition of RF was done according to the 3D definition from Image Biomarker Standardization Initiative [23]. Histogram based statistics were validated with the Medical Imaging Interaction Toolkit (MITK) [24]. The validation of geometric and texture features was done with an open source code [25]. For texture feature computation, SUV values of the voxels within the contour were discretized with a fixed bin width $(\mathrm{W}=0.01)[26,27]$, resulting in different numbers of bins (from 35 to 936, with average 237) depending on the range of SUV values in the contour. Texture features were derived from five matrices: the 3D version of the gray-level co-occurrence matrix (GLCM $[28,29])$; the gray-level run length matrix (GLRLM $[30,31])$, the gray-level size zone matrix (GLSZM [30, $32]$ ) and the neighborhood gray tone difference matrix (NGTDM [33]). In addition, on the voxel intensities within the contour we applied: (i) a Wavelet band-pass filtering (WF), with a weight ratio 1:2 between band-pass sub-bands and other sub-bands and (ii) an equal-probability quantization algorithm $(\mathrm{Q})$, by using the function histeq of MATLAB ${ }^{\circledR}$. The RF used in this study are listed in the Additional file 1: Table S1.

\section{Radiomics features selection: experimental phantom evaluation}

In order to avoid misinterpretation of the results derived from our investigation, experimental phantom evaluation was performed to select the RF that should be included in the different parts of our analysis. In the analysis of the prognostic value of FET-RF an experimental phantom evaluation was necessary to identify the RF robust to the different PET/CT systems employed in our cohorts. In the analysis of RF for tumor characterization, we compared volumes with different sizes, i.e. RF computed in tumor against RF computed in a 4 cc-sphere within the background. It was therefore necessary to identify the RF independent of the size of the volume (number of voxels within the contour). For the experimental evaluation we employed the PET images resulted from the EARL (ResEARch for Life - European Association of Nuclear Medicine initiative) accreditation measurement of the NEMA NU2 Phantom (NP). To analyze the robustness of RF for different PET/CT systems 18 contours were delineated: 12 spherical contours $(5.7-8.4 \mathrm{cc})$ were manually delineated in the background of NP and the 6 fillable spheres of the phantom $(0.5-25 \mathrm{cc})$ were segmented by applying a threshold of $40 \%$ of the maximum uptake within the sphere. To analyze the intrinsic dependency of RF with the volume 102 contours $(0.8-234 \mathrm{cc})$ were delineated in the background of the NP.

\section{Statistical analysis}

The statistical analysis was performed using in-house software based on Wolfram Mathematica v 11.2. Wilcoxon signed rank test was used when comparing two data samples. Correlations were analyzed in terms of Spearman's correlation tests and strong correlation was identified by $p<0.05$ and $r>0.8$. For TTP and OS analysis, Kaplan-Meier curves were estimated and comparison between groups was evaluated with the log-rank test. Multivariate Cox regression was used for estimation of hazard ratios (HR) with 95\% CI. For modelling with a binary output, an open source multivariate binary logistic regression analysis [25] was performed involving imbalance-adjusted bootstrap resampling (i.e. a resampling method alternative to the cross validation) in prediction performance estimation and computation of model coefficients. To correct for multiple test comparisons, the Bonferroni correction method was applied: the significance level was lowered to a value $p<\alpha / K$, where $\mathrm{K}$ is the number of comparisons and $\alpha$ is the significance level set to 0.05 .

\section{Results}

Radiomics features selection: experimental phantom evaluation

Results of the experimental phantom evaluation are summarized in the Additional file 2: Table S2. 61\% RF were robust (Wilcoxon signed rank test) to the different $\mathrm{PET} / \mathrm{CT}$ systems and 53\% RF had no strong correlation 
(Spearman's correlation test) with the number of voxels within the contour.

\section{FET radiomics features for tumor characterization}

RF computed on $\mathrm{V}_{\text {PETmax }}$ and $\mathrm{V}_{\text {PET3mm }}$ were compared with respect to the RF computed on the 4 cc-sphere defined as background in cerebrum $\left(\mathrm{V}_{\mathrm{Bg}}\right)$. The impact of the intrinsic dependency of some RF on the size was minimized by: (i) using the same size for $\mathrm{V}_{\mathrm{Bg}}$ in all patients and (ii) rejecting the RF with intrinsic dependence on size, based on results derived from an experimental phantom evaluation (see subsection Radiomics features selection: experimental phantom evaluation). Consequently, only the RF that did not show statistically significant strong correlation with the size were involved in the current analysis. 75\% of RF showed significant differences (Wilcoxon signed rank test, Bonferroni) between $\mathrm{V}_{\text {PETmax }}$ and $\mathrm{V}_{\mathrm{Bg}}$. For $\mathrm{V}_{\mathrm{PET} 3 \mathrm{~mm}}$, only $21 \%$ of RF had statistically different values with respect to $\mathrm{V}_{\mathrm{Bg}}$. Detailed results are listed in Additional file 2: Table S2.

\section{Added value of radiomics features with respect to conventional indices (SUV $\boldsymbol{m a x}_{\text {max }}$ and volume)}

For our patient cohort, we evaluated the added value of FET-RF with respect to the conventional indices $\mathrm{SUV}_{\text {max }}$ and volume. Overall, $64 \mathrm{RF}$ for $\mathrm{V}_{\mathrm{PETmax}}$ and $60 \mathrm{RF}$ for $\mathrm{V}_{\text {PET3mm }}$ were simultaneously independent of volume and $\mathrm{SUV}_{\max }$. Results are presented in Additional file 2: Table S2.

\section{Prognostic value of FET radiomics features} Univariate analysis

To avoid misinterpretation of the results, only RF that have been proven in the phantom evaluation to be robust to the different PET/CT scanners were included (see subsections Radiomics features selection: experimental phantom evaluation). In Table 2 the RF with the best Kaplan-Meier curve performance (log-rank test: $p<0.05)$ are presented. Overall, only the texture feature SmallZone-Low-Gray-Emphasis (SZGLE) could predict earlier time-to-tumor progression (TTP) when the significance level was lowered by the Bonferroni multiple-test correction (statistically significant for $p<0.0013$ ).

\section{Multivariate analysis: radiomics-signatures}

To build radiomics-signatures, i.e. combinations of RF for the prognosis of OS and for TTP, we focused our analysis on the RF presented in Table 2, i.e. the RF robust to the PET/CT scanner and with the best Kaplan-Meier performance for the univariate analysis. To remove redundancy, we assessed the correlations between these RF. From each of the resulted correlated-feature groups, only the RF with more statistically significant prediction (lower $p$ value in log-rank test), was selected. These RF were combined (radiomics-signature) into a multivariate Cox proportional hazards regression model for prediction of OS and of TTP. The resulting radiomics-signatures consisted:

Table 2 Radiomics features derived from the two contours $\left(V_{P E T m a x}\right.$ and $\left.V_{\text {PET3mm }}\right)$ showing significant $p$ values $<0.05$ in the Log-rank test for the Kaplan-Meier curves of overall-survival (OS) and time-to-tumor-progression (TTP)

\begin{tabular}{|c|c|c|c|c|}
\hline & & $\begin{array}{l}\text { Correlation with overall survival } \\
(\mathrm{OS}): p \text { value }\end{array}$ & & $\begin{array}{l}\text { Correlation with time-to- } \\
\text { progression (TTP): } p \text { value }\end{array}$ \\
\hline \multirow{4}{*}{$\begin{array}{l}\text { Radiomics features } \\
\text { extracted from } V_{\text {PETmax }}\end{array}$} & \multirow[t]{4}{*}{$S U V_{\min }$} & 0.038 & SZLGE & 0.001 \\
\hline & & & Busyness & 0.006 \\
\hline & & & WF_TS & 0.012 \\
\hline & & & QVariance $_{\mathrm{CM}}$ & 0.029 \\
\hline \multirow{11}{*}{$\begin{array}{l}\text { Radiomics features } \\
\text { extracted from } V_{\text {PET3mm }}\end{array}$} & $S U V_{\text {mean }}$ & 0.041 & \multirow[t]{11}{*}{ Eccentricity } & \multirow[t]{11}{*}{0.004} \\
\hline & GLV & 0.033 & & \\
\hline & GLV2 & 0.011 & & \\
\hline & WF_GLV & 0.002 & & \\
\hline & QAcor & 0.013 & & \\
\hline & QHGZE & 0.013 & & \\
\hline & QSZHGE & 0.013 & & \\
\hline & QGLN2 & 0.033 & & \\
\hline & QHGRE & 0.008 & & \\
\hline & QSRHGE & 0.008 & & \\
\hline & QLRHGE & 0.008 & & \\
\hline
\end{tabular}


(i) for the prediction of OS (OS-radiomics-signature) in the combination of $\mathrm{SUV}_{\text {mean }}, \mathrm{WF}$ GLV and QLRHGE for $\mathrm{V}_{\text {PET3mm }}$ and $\mathrm{SUV}_{\min }$ for $\mathrm{V}_{\text {PETmax }}$

(ii) for the prediction of TTP (TTP-radiomics-signature) in the combination of SZLGE, Busyness and QVariance ${ }_{\mathrm{CM}}$ for $\mathrm{V}_{\mathrm{PETmax}}$ and Eccentricity for $\mathrm{V}_{\text {PET3mm }}$.

The $p$ value for all three overall tests (Likelihood-Ratio, Wald, and Score) was significant $(p<0.05)$ for both radiomics-signature models. In Fig. 2 the Kaplan-Meier curves and log-rank test for the resulted radiomics-signature models are shown. For the TTP-radiomics-signature, results from the Kaplan-Meier curve and log-rank test (Fig. 2b) showed the same performance as when only considering the texture feature SZLGE.

For TTP-radiomics-signature and SZLGE we additionally evaluated their power as predictors of RL. Results showed moderate RL predictions with an area-under-the-curve (AUC) and sensitivity of 0.66 and 0.78 for the TTP-radiomics-signature and 0.63 and 0.79 for SZLGE, respectively. Therefore, for TTP-signature and for SZLGE, high values indicated not only earlier progression (TTP), but also a RL within the initial FET-PET active volume (Fig. 3).

\section{Discussion}

To our knowledge, this is the first evaluation of the prognostic role of FET-RF in patients with rGBM scheduled for re-irradiation. The feasibility of developing a prognostic model based on FET-RF could be demonstrated, with the texture feature Small-Zone-Low-Grey-Emphasis (SZLGE) showing the best performance for the prediction of time-to-progression (TTP) and in field versus distant RL. Considering that re-irradiation can be associated with side effects like radiation necrosis, brain edema, increased corticosteroid use or need for bevacizumab treatment, these results could have a significant clinical importance in the selection of suitable patients. These results are to be further validated in larger and multi-institutional patient cohorts (e.g. patients in the multicenter GLIAA trial [21]) and could therefore be considered for the development of future prognostic scores.

In the past decade, the number of radiomics publications has dramatically increased. However, few articles have properly addressed the unique challenges that a model development based on PET-radiomics may pose, as a result of the dependence of the RF variability on multiple factors. In the following, we detailed the main considerations applied in the methodology of this study in order to minimize redundancy and to maximize robustness of RF, as recommended in previous PET-radiomics guideline publications [34-37]. Firstly, the definition of RF was performed according to the Image Biomarker Standardization
Initiative [23] and recommendations for resampling and 3 D definition [26, 27] were followed in order to minimize the impact on RF variability caused by the definition of texture feature matrices [29]. Additionally, our in-house code was validated with the open source software MITK [24] and an open source MATLAB code [25]. It permits to easily reproduce the computation of the RF involved in our study. Secondly, the impact of different reconstruction parameters and scanner design on PET RF variability [38] was assessed with experimental phantom evaluation. The intrinsic dependence of some RF on the statistics implied by the number of voxels within the volume [36] was also evaluated with experimental phantoms. Consequently, only the RF robust with respect to the different PET/CT scanners and independent of the number of voxels within the contour were involved in the development of the prognosis model and in the evaluation of the tumor characterization, respectively. Thirdly, in order to minimize the well-known inter- and intra-user variability observed by manual contouring and therefore its impact on RF variability [26, 34], a semi-automatic segmentation algorithm was employed. In addition, the added value of the RF (not redundancy) with respect to the parameters conventionally used for tumor characterization (volume and $\mathrm{SUV}_{\max }$ ) was assessed. Results showed that texture features provided complementary information with respect to these conventional parameters and therefore their evaluation in the design of prognostic models could be supported. Finally, the generalizability of the results has been evaluated by internal validation, using imbalance-adjusted bootstrap as a resampling strategy in model development [25] and a strong criterion for multiple test correction (Bonferroni) was applied in order to reject false positives.

Conventionally, radiomics for brain tumors has primarily focused on MRI. However, recent publications have also evaluated the use of FET-PET radiomics for differentiation of grade III and IV primary tumors [18], the diagnosis of pseudoprogression in high-grade glioma $[19,39]$ and the correlation with isocitrate dehydrogenase genotype [20]. In agreement with previous publications, results of our investigation confirmed that FET-PET can have a prognostic value in the field of neuro-oncology [6-14, 40]. We presented the first evaluation of FET-PET radiomics for the prognosis assessment of rGBM patients scheduled for reirradiation. Although the size of our cohort was limited by its prospective character, it was comparable or larger than the cohorts evaluated in previous publications $[36,39]$.

The texture feature that showed the most statistically significant prognostic value was SZLGE derived from the high-risk volume $V_{\text {PETmax }}$. More precisely, high values of SZLGE and of the TTP-radiomics-signature in the FET-PET tumor volume before re-irradiation were correlated with earlier recurrence and a localization of the 


\section{Multivariate Cox proportional hazards regression model}

a Overall Survival (OS)

Statistic P-Value

Log-Rank $4.30647 \quad 0.0379676$

Probability

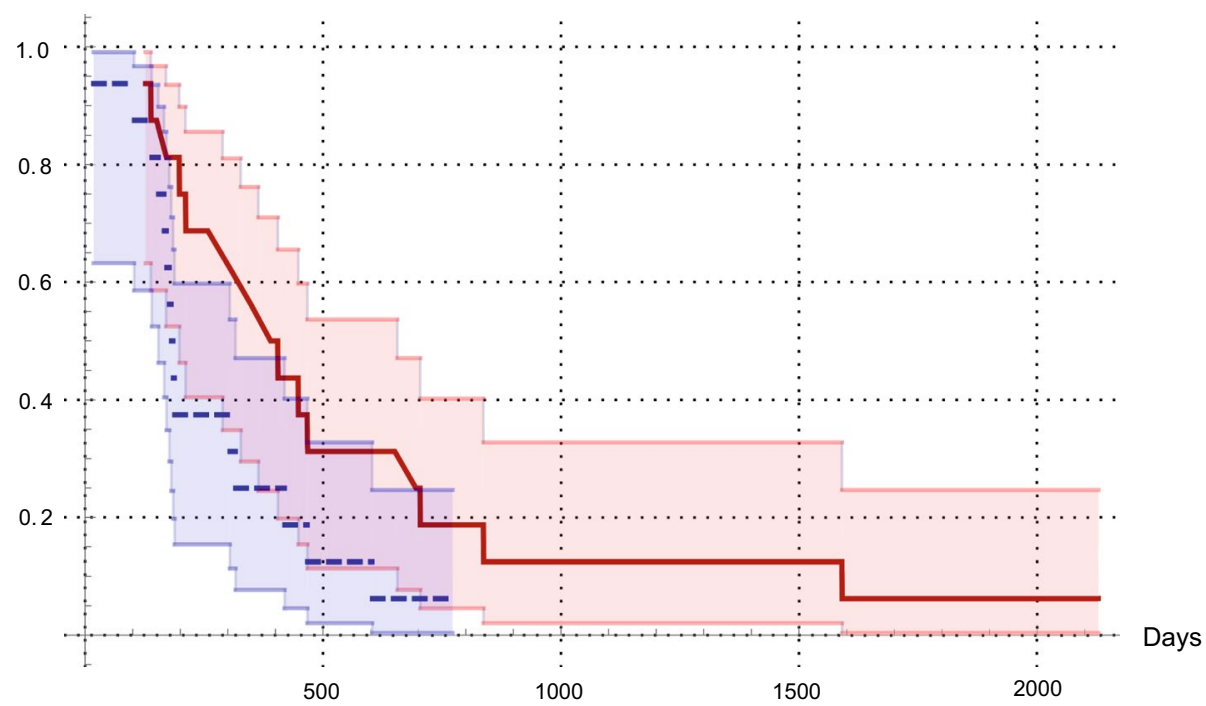

- Signature ४Median - - - - Signature >Median

b Time - to-Progression (TTP)

Statistic P-Value

\begin{tabular}{l|ll}
\hline Log-Rank & 10.58430 .00114049
\end{tabular}

Probability

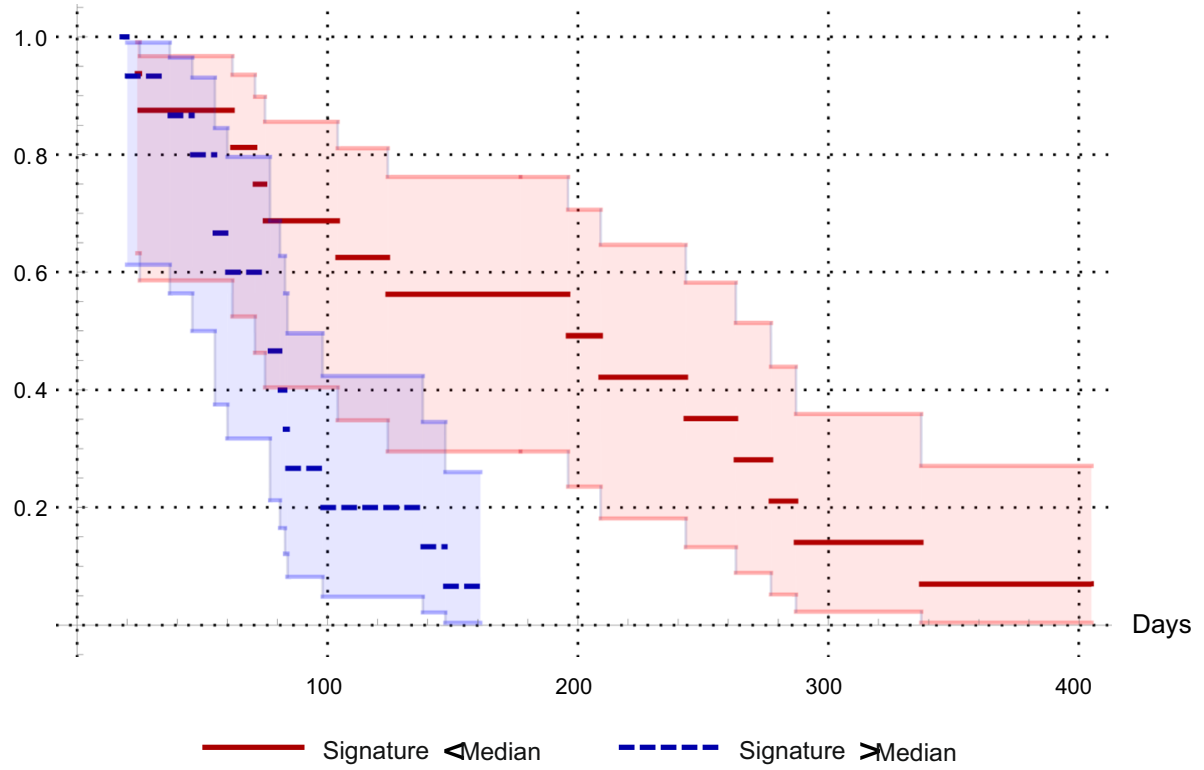

Fig. 2 Kaplan-Meier curves and log-rank test for the radiomics-signatures predicting overall survival (OS-radiomics-signature) (a) and time-to-progression (TTP-radiomics-signature) $(\mathbf{b})$ 


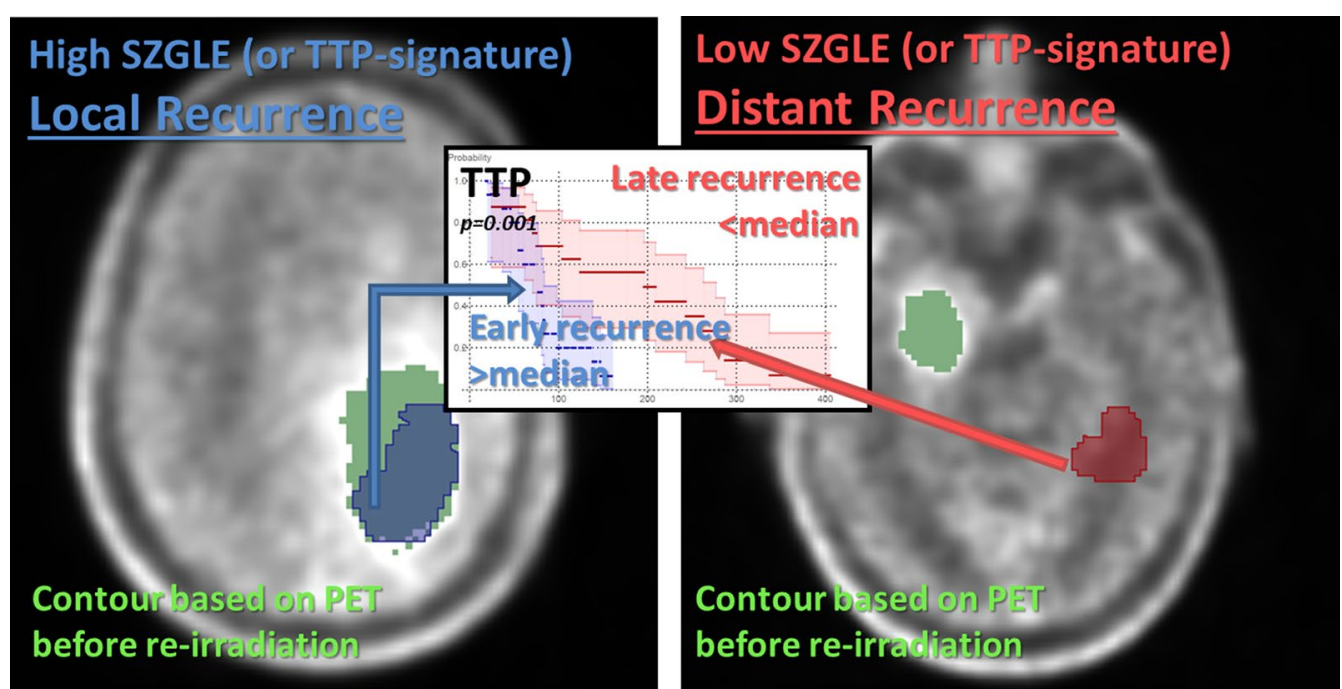

Fig. 3 Scheme of the pattern observed for values of SZLGE and TTP-radiomics-signature in regards to the recurrence location (AUC SZLGE $=0.63$ and $\mathrm{AUC}_{\text {TTP-radiomics-signature }}=0.66$ ) and time-to-progression (Kaplan-Meier curves $\mathrm{P}_{\text {SZLGE }}=\mathrm{P}_{\text {TTP-radiomics-signature }}=0.001$ ): high SZGLE for early in field recurrence after re-RT (blue) and low SZGLE for late distant recurrence after re-RT (red)

recurrence within the gross-tumor-volume defined on PET. These patients would therefore probably benefit less from re-irradiation and could be considered for alternative therapies (surgery, chemotherapy) or irradiation dose escalation. Low values of SZLGE and of the TTP-radiomics-signature correlated with longer TTP and distant recurrences, which is consistent with previous publications showing longer progression-free survival in patients with non-local recurrences [41]. Furthermore, we also identified an OS-radiomics-signature with statistical significance ( $p=0.038$, Fig. 2).

From the two FET-PET quantification parameters with prognostic value (SZGLE and the TTP-radiomics-signature), SZGLE should be preferred because of its easier computation. In addition, SZGLE has been previously reported to be robust with respect to analogical/digital PET/CT systems, CT artefacts, segmentation methods and lesion motion $[26,42]$. Consequently, the reproducibility of SZLGE for the PET/CT imaging protocols conveyed by different institutions could be expected. SZLGE quantifies the amount and the signal intensity of small zones of low uptake. SZLGE prediction of TTP and RL and its statistically significant discrimination between tumor and background suggested that small low FET-uptake regions may also play an important role in the characterization of aggressive tumors. In addition, SZLGE is derived from the gray-level size zone matrix (GLSZM [30, 32]), which has been previously reported to provide quantitative parameters for non-invasive prediction of the IDH genotype in gliomas [20].
The main limitation of our study was that our results could not be validated on a second patient cohort with similar characteristics. We attempted an internal validation by retrospective selection of rGBM patients treated at our institution. We were able to collect 22 patients who underwent the same FET-PET acquisition protocol, but clinical characteristics differed significantly from the original cohort: IDH status, use of surgery, delivered dose, follow-up time, percentage of patients with recurrence, etc. Consequently, this cohort was considered not appropriate for the validation of our findings and future validation in a larger prospective cohort with similar clinical characteristics is still required. However, it is worth mentioning that for the patients of the retrospective cohort with recurrence (45\%), values for SZLGE and the TTP-radiomics-signature confirmed the pattern observed in the initial cohort (Fig. 3) and significance was achieved for the prediction of RL. Specifically, patients with distant recurrence presented low SZLGE (average $0.019<$ median) and late progression (average 155 days, with 180 days for the initial cohort), while patients with local recurrence showed high SZLGE (average $0.035>$ median) and early progression (average 94 days, with 77 days for the initial cohort). Furthermore, the TTP-radiomics-signature (and SZGLE) predicted RL with AUC $=0.68(0.58)$ and sensitivity $=0.79(0.84)$.

Future work will involve all the patients prospectively enrolled in the multicenter GLIAA trial [21] and will therefore allow an additional external validation of our preliminary results. After confirmation, SZLGE could 
therefore be suggested for the improvement of individualized patient and treatment selection in rGBM.

\section{Conclusions}

We presented the first evaluation of the role of FETPET RF in the prognosis of patients with rGBM scheduled for re-irradiation. Our findings have shown that the radiomic feature SZLGE and two TTP- and OSradiomics-signatures could significantly distinguish reirradiation responders from non-responders. Further analysis in a larger prospective validation cohort is warranted and planned.

\section{Supplementary information}

The online version contains supplementary material available at https://doi. org/10.1186/s13014-020-01744-8.

\section{Additional file 1. Table of radiomics features.}

Additional file 2. Results of radiomics features analysis. Black box means positive result for the analysis described on the first row and represents the property of interest; like for example, RF robust to the different PET/CT systems (second column).

\section{Abbreviations}

FET: O-(2-[18F]fluoroethyl)-L-tyrosine; PET: Positron emission tomography; rGBM: Recurrent glioblastoma; RF: Radiomics features; SZLGE: Small-ZoneLow-Gray-Level-Emphasis; TTP: Time-to-progression; LR: Local Recurrence; DM: Distant Metastasis; CT: Computed tomography; SUV: Standardized uptake value; SD: Standard deviation; RT: Radiation therapy; TF64: GEMINI TF TOF 64; BB: GEMINI TF 16 Big Bore; EANM: European Association of Nuclear Medicine; EARL: European Association Research Ltd; BLOB-OS-TF: LOR-based ordered-subset iterative time-of-flight algorithm using spherical coordinates; HR: Hazard ratios; re-RT: Re-irradiation; MRI: Magnetic resonance imaging; Gd-T1MR: Gadolinium contrast enhanced T1-weighted images; MITK: Medical Imaging Interaction Toolkit; WF: Wavelet band-pass filtering; Q: Quantization algorithm; NP: NEMA NU2 Phantom; AUC: Area-under-the-curve.

\section{Authors' contibutions}

Recruitment, pre-processing, design, analysis and writing: MC and IP. Preprocessing: MS. Recruitment: MM, DB and ALG. Clinical Information: TS and ALG. Manuscript review and discussion: HU, FE, MM, MN, DB and ALG. All authors read and approved the final manuscript.

\section{Fundings}

Open Access funding enabled and organized by Projekt DEAL. Montserrat Carles was partially funded in 2019 and 2020 by Era PerMed and funded from July 2020 on by the Conselleria de Sanitat Universal i Salut Pública from the Comunitat Valenciana. Ilinca Popp was partially funded by the Else KrönerFresenius-Stiftung, Germany, within the EXCEL (Excellent Clinician Scientists in Freiburg - Education for Leadership) Program "Immunological Causes and Therapies of Cancer". Franziska Eckert was partly funded by the Else-KroenerFresenius Research Foundation under Grant 2015_Kolleg.14. The funding sources had no involvement in the writing of the manuscript or in the decision to submit the article for publication.

\section{Availability of data and materials}

The datasets during and/or analysed during the current study available from the corresponding author on reasonable request.

\section{Ethics approval and consent to participate}

All procedures performed in studies involving human participants were in accordance with the ethical standards of the institutional and/or national research committee and with the 1964 Helsinki declaration and its later amendments or comparable ethical standards.

\section{Consent for publication}

Informed consent was obtained from all individual participants included in the study.

\section{Competing interests}

Franziska Eckert has aresearch collaboration with Merck KgAa as well as research and educational grants from Elekta, Philips, Siemens, Sennewald. Franziska Eckert declares that she has no conflict of interest. All authors declare no conflict of interest.

\section{Author details}

1 Division of Medical Physics, Department of Radiation Oncology, Medical Center, University of Freiburg, Robert-Koch Str. 3, 79106 Freiburg, Germany. ${ }^{2}$ German Cancer Consortium (DKTK), German Cancer Research Center (DKFZ), Partner Site Freiburg, Heidelberg, Germany. ${ }^{3}$ Biomedical Imaging Research Group (GIBI230-PREBI), Imaging La Fe Node at Distributed Network for Biomedical Imaging (ReDIB) Unique Scientific and Technical Infrastructures (ICTS), La Fe Health Research Institute, Valencia, Spain. ${ }^{4}$ Department of Radiation Oncology, Medical Center, Faculty of Medicine, University of Freiburg, Freiburg, Germany. ${ }^{5}$ Department of Nuclear Medicine, Medical Center, Faculty of Medicine, University of Freiburg, Freiburg, Germany. ${ }^{6}$ Department of Neuroradiology, Medical Center, Faculty of Medicine, University of Freiburg, Freiburg, Germany. ${ }^{7}$ Department of Radiation Oncology, University Hospital Tübingen, Tübingen, Germany. ${ }^{8}$ German Cancer Consortium (DKTK), German Cancer Research Center (DKFZ), Partner Site Tübingen, Tübingen, Germany. ${ }^{9}$ Department of Radiation Oncology, University Hospital, LMU Munich, Munich, Germany. ${ }^{10}$ Cerman Cancer Consortium (DKTK), German Cancer Research Center (DKFZ), Partner Site Munich, Munich, Germany.

Received: 9 July 2020 Accepted: 29 December 2020

Published online: 03 March 2021

\section{References}

1. Stupp R, Mason WP, van den Bent MJ, Weller M, Fisher B, Taphoorn MJB, et al. Radiotherapy plus concomitant and adjuvant temozolomide for glioblastoma. N Engl J Med. 2005;352(10):987-96.

2. Nieder C, Astner ST, Mehta MP, Grosu AL, Molls M. Improvement, clinical course, and quality of life after palliative radiotherapy for recurrent glioblastoma. Am J Clin Oncol. 2008;31(3):300-5.

3. Barney C, Shukla G, Bhamidipati D, Palmer JD. Re-irradiation for recurrent glioblastoma multiforme. Chin Clin Oncol. 2017;6(4):36. https://doi. org/10.21037/cco.2017.06.18.

4. Niyazi M, Adeberg S, Kaul D, Boulesteix A-L, Bougatf N, Fleischmann DF, et al. Independent validation of a new reirradiation risk score (RRRS) for glioma patients predicting post-recurrence survival: a multicenter DKTK/ ROG analysis. Radiother Oncol. 2018;127(1):121-7.

5. Combs SE, Niyazi M, Adeberg S, et al. Re-irradiation of recurrent gliomas: pooled analysis and validation of an established prognostic score-report of the Radiation Oncology Group (ROG) of the German Cancer Consortium (DKTK). Cancer Med. 2018;7(5):1742-9.

6. Fleischmann DF, et al. (18)F-FET PET prior to recurrent high-grade glioma re-irradiation-additional prognostic value of dynamic time-to-peak analysis and early static summation images? J Neuroonol. 2017;132(2):277-86.

7. Moller $\mathrm{S}$, et al. Prognostic value of 18 F-FET PET imaging in re-irradiation of high-grade glioma: results of a phase I clinical trial. Radiother Oncol. 2016;121(1):132-7.

8. Niyazi M, Jansen N, Ganswindt U, et al. Re-irradiation in recurrent malignant glioma: prognostic value of [18F]FET-PET. J Neurooncol. 2012;110(3):389-95.

9. Grosu AL, Weber WA. PET for radiation treatment planning of brain tumors. Radiother Oncol. 2010;96:325-7.

10. Galldiks N, DunkI V, Stoffels G, Hutterer M, Rapp M, Sabel M, et al. Diagnosis of pseudoprogression in patients with glioblastoma using O-(2-[18F]fluoroethyl)-L-tyrosine PET. Eur J Nucl Med Mol Imaging. 2015;42(5):685-95. 
11. Grosu AL, Astner ST, Riedel E, Nieder C, Wiedenmann N, Heinemann F, et al. An interindividual comparison of O-(2)-18F-Fluoroethyl-L-Tyrosine (FET) and L-Methyl-11C-Methionine-PET in patients with brain gliomas and metastases. Int J Radiat Oncol Biol Phys. 2011;81(4):1049-58.

12. Galldiks N, Stoffels G, Filss C, Rapp M, Blau T, Tscherpel C, et al. The use of dynamic O-(2-18F-fluoroethyl)-l-tyrosine PET in the diagnosis of patients with progressive and recurrent glioma. Neuro-oncology. 2015;17(9):1293-300.

13. Grosu A-L, Weber WA, Riedel E, Jeremic B, Nieder C, Franz M, et al. L-(methyl-11C) methionine positron emission tomography for target delineation in resected high-grade gliomas before radiotherapy. Int J Radiat Oncol Biol Phys. 2005;63(1):64-74

14. Niyazi M, Geisler J, Siefert A, Schwarz SB, Ganswindt U, Garny S, et al. FET-PET for malignant glioma treatment planning. Radiother Oncol. 2011;99(1):44-8.

15. Gilles RJ, Kinahan PE, Hricak H. Images are more than pictures, they are data. Radiology. 2016;278(2):563-77.

16. Avanzo M, Stancanello J, El Naqa I. Beyond imaging: the promise of radiomics. Phys Med. 2017;38:122-39.

17. Macyszyn L, Akbari H, Pisapia JM, Da X, Attiah M, Pigrish V, et al. Imaging patterns predict patient survival and molecular subtype in glioblastoma via machine learning techniques. Neuro Oncol. 2016;18(3):417-25.

18. Kickingereder P, Neuberger U, Bonekamp D, Piechotta PL, Götz M, Wick $A$, et al. Radiomic subtyping improves disease stratification beyond key molecular, clinical, and standard imaging characteristics in patients with glioblastoma. Neuro-oncology. 2018;20(6):848-57.

19. Pyka T, Gempt J, Hiob D, Ringel F, Schlegel J, Bette S, et al. Textural analysis of pre-therapeutic [18F]-FET-PET and its correlation with tumor grade and patient survival in high-grade gliomas. Eur J Nucl Med Mol Imaging 2016;43(1):133-41.

20. Lohmann P, Lerche C, Bauer EK, Steger J, Stoffels G, Blau T, et al. Predicting IDH genotype in gliomas using FET PET radiomics. Sci Rep. 2018;8(1):13328.

21. Oehlke O, Mix M, Graf E, Schimek-Jasch T, Nestle U, Götz I, et al. Aminoacid PET versus MRI guided re-irradiation in patients with recurrent glioblastoma multiforme (GLIAA) - protocol of a randomized phase II trial (NOA 10/ARO 2013-1). BMC Cancer. 2016;16(1):769.

22. Wen PY, Macdonald DR, Reardon DA, et al. Updated response assessment criteria for high-grade gliomas: response assessment in neuro-oncology working group. J Clin Oncol. 2010;28(11):1963-72.

23. Zwanenburg A, Leger S, Vallières M, Löck S. The Image Biomarker Standardization Initiative: standardized quantitative radiomics for highthroughput image-based phenotyping. Radiology. 2020;295(2):328-38.

24. Wolf I, Vetter $M$, Wegner I, Böttger T, Nolden $M$, Schöbinger $M$, et al. The medical imaging interaction toolkit. Med Image Anal. 2005;9(6):594-604.

25. Vallières M, Freeman CR, Skamene SR, El Naqa I. A radiomics model from joint FDG-PET and MRI texture features for the prediction of lung metastases in soft-tissue sarcomas of the extremities. Phys Med Biol. 2015;60(14):5471-96.

26. Carles M, Bach T, Torres-Espallardo I, Baltas D, Nestle U, Martí-Bonmatí L. Significance of the impact of motion compensation on the variability of PET image features. Phys Med Biol 2018 21;63(6):065013.

27. Leijenaar RTH, Nalbantov G, Carvalho S, van Elmpt WJC, Troost EGC, Boellaard R, et al. The effect of SUV discretization in quantitative FDG-PET radiomics: the need for standardized methodology in tumor texture analysis. Sci Rep. 2015;5:11075.
28. El Naqa I, Grigsby P, Apte A, Kidd E, Donnelly E, Khullar D, et al. Exploring feature-based approaches in PET images for predicting cancer treatment outcomes. Pattern Recognit. 2009;42(6):1162-71.

29. Hatt M, Majdoub M, Vallières M, Tixier F, Le Rest CC, Groheux D, et al. 18FFDG PET uptake characterization through texture analysis: investigating the complementary nature of heterogeneity and functional tumor volume in a multi-cancer site patient cohort. J Nucl Med. 2015;56(1):38-44.

30. Xu D-H et al. Run-length encoding for volumetric texture. In: The 4th IASTED international conference on visualization, imaging, and image processing. 2004.

31. Chu A, Sehgal CM, Greenleaf JF. Use of gray value distribution of run lengths for texture analysis. Pattern Recognit Lett. 1990;11(6):415-9.

32. Sassi OB, Sellami L, Slima MB, Chtourou K, Hamida AB. Improved spatial gray level dependence matrices for texture analysis. Int J Comput Sci Inf Technol (IJCSIT). 2012;4(6):209-19.

33. Amadasun M, King R. Textural features corresponding to textural properties. IEEE Trans Syst Man Cybern. 1989;19:1264-74.

34. Reuze $\mathrm{S}$, et al. Radiomics in nuclear medicine applied to radiation therapy: methods, pitfalls, and challenges. Int J Radiat Oncol Biol Phys. 2018;102(4):1117-42.

35. Zwanenburg A. Radiomics in nuclear medicine: robustness, reproducibility, standardization, and how to avoid data analysis traps and replication crisis. Eur J Nucl Med Mol Imaging. 2019;46:2638-55. https://doi. org/10.1007/s00259-019-04391-8.

36. Hatt M, Tixier F, Pierce L, Kinahan PE, Le Rest CC, Visvikis D. Characterization of PET/CT images using texture analysis: the past, the present... any future? Eur J Nucl Med Mol Imaging. 2017;44(1):151-65.

37. Varghese BA. Texture analysis of imaging: what radiologists need to know. 2019;212(3):520-8. https://doi.org/10.2214/AJR.18.20624.

38. Yan J, Chu-Shern JL, Loi HY, Khor LK, Sinha AK, Quek ST, et al. Impact of image reconstruction settings on texture features in 18F-FDG PET. J Nucl Med. 2015;56(11):1667-73.

39. Kebir S, Khurshid Z, Gaertner FC, Essler M, Hattingen E, Fimmers R, et al. Unsupervised consensus cluster analysis of $\left[{ }^{18} \mathrm{~F}\right]$-fluoroethyl-L-tyrosine positron emission tomography identified textural features for the diagnosis of pseudoprogression in high-grade glioma. Oncotarget. 2017;8(5):8294-304.

40. Suchorska B, Jansen NL, Linn J, Kretzschmar H, Janssen H, Eigenbrod S, et al. Biological tumor volume in ${ }^{18} \mathrm{FET}$-PET before radiochemotherapy correlates with survival in GBM. Neurology. 2015;84(7):710-9.

41. Tejada S, Díez-Valle R, Aldave G, Marigil M, de Gallego J, Domínguez PD. Factors associated with a higher rate of distant failure after primary treatment for glioblastoma. J Neurooncol. 2014;116(1):169-75.

42. Carles, et al. PET Image features for their use in Radiomics: selection criteria based on intrinsic complementarity with respect to volume and robustness with respect to system, metal artefacts and voxel size in image reconstruction. Nuklearmedizin. 2019;58(02):171. https://doi. org/10.1055/s-0039-1683671.

\section{Publisher's Note}

Springer Nature remains neutral with regard to jurisdictional claims in published maps and institutional affiliations. 